\title{
IoT deployment for city scale air quality monitoring with Low-Power Wide Area Networks
}

\author{
Steven J. Johnston*† Philip J. Basford* Florentin M. J. Bulot* Mihaela Apetroaie-Cristea* \\ Gavin L Foster ${ }^{\S}$ Matthew Loxham ${ }^{\ddagger}$ Simon J. Cox* \\ ${ }^{*}$ Faculty of Engineering and the Environment $/ \S$ National Oceanography Centre/ ${ }^{\ddagger}$ Faculty of Medicine, \\ University of Southampton, Southampton, UK. \\ $\dagger$ sjj698@zepler.org
}

\begin{abstract}
Air Quality (AQ) is a very topical issue for many cities and has a direct impact on the health of its citizens. We propose to investigate the air quality of a large UK city using low-cost Particulate Matter (PM) micro-sensors, and compare them with government operated air quality stations. In this pilot deployment we design and build six AQ IoT devices, each with four different low-cost PM sensors and deploy them at two locations within the city. These devices are equipped with LoRaWAN wireless network transceivers to test city scale Low-Power WideArea Network network coverage. We conclude that some low-cost $P M$ sensors are viable for monitoring $A Q$ and demonstrate that our device design can be used via LoRaWAN to facilitate more granular city coverage without limitations of network access. Based on these findings we intend to deploy a larger LoRaWAN enabled Air Quality sensor network deployment across the city.
\end{abstract}

Keywords-Internet of Things, Wireless Sensor Networks, Air quality, Urban Pollution.

\section{INTRODUCTION}

Six Internet of Things (IoT) devices for monitoring air quality have been deployed across two schools in Southampton, UK. This deployment is a pilot project and if successful will be expanded to include more sites within the city. The objective of this pilot project is to demonstrate the capability of the AQ IoT devices to capture spatio-temporal variations of air pollutants in order to raise awareness of air quality issues amongst the public. These devices also act as a feasibility study for low-power long range wireless communication (LoRaWAN) to enable future sites without existing network connectivity to be monitored. Existing AQ sensing networks use a wider range of sensors and do not use Low-Power networks, but the hardware is considerably more expensive, for example CAIRSENSE [1], OpenSense [2] and Citi-Sense-MOB [3]. We show preliminary PM sensor data and correlate it with reference air quality stations within the city. The findings from this pilot deployment are positive and demonstrate that lowcost PM sensors are viable and that city wide long range wireless technologies can play a role in sensor networks.

\section{AIR QUALITY MONITORING}

Air pollution exerts a major burden on health worldwide[4] being responsible for 6.5 million premature deaths in 2015[5]. Air pollutant concentrations and personal exposure levels vary extensively between different indoor and outdoor environments. There is also variation in concentrations at a fine spatio-temporal scale in urban environments[6], [7], [8]. Particulate matter (PM) of relevance to impact on health has an aerodynamic diameter lower than $10 \mu \mathrm{m}$, although the most strongly linked effects are to fine $\mathrm{PM}, \mathrm{PM}_{2.5}$, with aerodynamic diameter lower than $2.5 \mu \mathrm{m}$. PM can cause a wide range of adverse effects on humans even at low concentrations[9]. In the UK, exposure to $\mathrm{PM}_{2.5}$ is responsible for 29,000 deaths per year with an uncertainty of $75 \%$ [4]. This wide confidence interval results from the fact that the impact of exposure to air pollution on health cannot be captured accurately simply by the concentrations of the pollutant because it depends also on the duration and the frequency of the exposure[10]. At individual level, various other parameters, including age and health status, also play roles. There is currently a poor ability to determine personal exposure to pollution, given the lack of pollution monitoring stations, and fine spatio-temporal resolution of pollution variation. High spatio-temporal coverage of pollutants measurements is therefore urgently needed to improve our understanding of air pollutant sources and exposure.

At a national level in the UK, PM are monitored by the Automatic Urban and Rural Network (AURN) stations[11]. These stations provide reliable and robust data about background concentration levels but are expensive, and require significant expertise to maintain. This makes it difficult to attain the high spatial resolution [12] required to better assess personal exposure and to precisely identify pollution sources. Different approaches need to be combined to solve this issue[13].

Several projects to establish innovative air monitoring networks have been conducted in cities in Europe and in the USA using low-cost micro-sensors. Some use mobile measurements mounted on cars[6], on trams[14], on bikes[15], [3] or on pedestrians[16]. Although low-cost micro-sensors offer a means to increase the granularity of the data available, the extent to which they can provide valid data first needs to be evaluated. Low-cost micro-sensors may be used to complement existing air pollution monitoring networks by providing the spatio-temporal resolution required to improve our understanding of air pollutants and our exposure to them[17].

The deployment of a dense, accurate, reliable city-wide network of PM sensors could improve our ability to identify sources of pollution, understand personal exposure and complement existing monitoring networks to raise awareness 


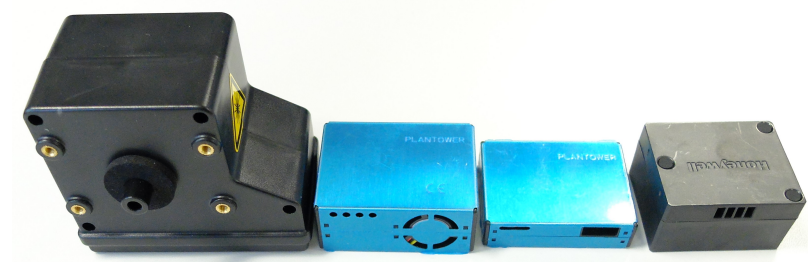

Fig. 1. Particulate matter sensors Alphasense OPC-N2, Plantower PMS5003, Plantower PMS7003, Honeywell HPMA115S0

among the population.

\section{PARTICUlate MATTER SENSORS}

Low-cost micro-sensors are already being used to monitor PM pollution (see Figure 1). Their prices range from a few USD to a few hundred USD making it possible to deploy dense city-wide networks. These micro-sensors need to be plugged into a processor (e.g. Raspberry $\mathrm{Pi}$ ), equipped with the means to communicate or store the data collected. The most common low-cost PM micro-sensors are Optical Particle Counters (OPC), based on light-scattering. They can typically detect particles with aerodynamic diameters ranging from $0.3 \mu \mathrm{m}$ to $10 \mu \mathrm{m}[18]$. Below $0.3 \mu \mathrm{m}$ the particles do not scatter light sufficiently and over $10 \mu \mathrm{m}$, depending on the actual size of the inlet, they cannot enter the sensor. These sensors transform the signal measured into a raw particulate count or/and mass concentration. The lower limit of detection of these sensors is generally between 1 to $10 \mu \mathrm{g} / \mathrm{m}^{3}$ which is the same order of magnitude as the World Health Organisation annual mean guideline for $\mathrm{PM}_{2.5}$ [19]. Reference methods for measuring PM mass concentration rely on the direct determination of particle mass, rather than inference of particle mass from particle count. Mass concentration is used in the legislation and is necessary to compare reference measurement instruments, but the raw particulate count is also useful, especially at concentration levels close to the sensor limit.

The key drawback of using low-cost PM micro-sensors is the data quality, which may be susceptible to: (i) drift over time[1], [20], (ii) interference from climate conditions, (iii) differing performance in differing environments, (iv) a lack of reproducibility between sensor units, (v) the composition of the PM[21].

Different models of PM micro-sensors may present different characteristics and will behave differently regarding the data quality issues listed above. In order to address this issue, four PM micro-sensors have been selected for this deployment based on their popularity in the literature, on their ease of use or on their availability:(i) Alphasense OPC-N2, (ii) Plantower PMS5003, (iii) Plantower PMS7003, (iv) Honeywell HPMA115S0 (from left to right on Figure 1). The main characteristics of the sensors are listed in Table I.

\section{IOT DEVICE}

\section{A. Hardware}

The air quality IoT Device is built around the Raspberry Pi 3 Model B, which is then equipped with a Power Over Ethernet
(PoE)[26] HAT in order to enable low voltage power and network connectivity. The power is provided using a standalone PoE injector. A LoRaWAN HAT is also installed to provide a secondary communication method which is discussed further in Section V. The LoRaWAN HAT also provides a GPS receiver connected to the on-board serial port, this is currently only used to set the system time (RTC), however, in the future it can be used for mobile applications. Each node is also equipped with temperature and relative humidity monitoring as relative humidity, and temperature to a lesser extent, are potential data confounders for the particulate readings of some PM micro-sensors[27], [28].

Three of the PM sensors require a serial port and the LoRaWAN HAT uses the Raspberry Pis single serial port for the GPS device. FTDI USB-serial converters were used to add serial ports, in particular because each device has a unique serial number. This serial number enables consistent naming of serial ports between reboots, something which cannot be guaranteed with all such devices. The Raspberry Pis onboard SPI device is used to interface with the LoRaWAN module, but testing showed that repeatable reliable behaviour could not be achieved using this with the SPI OPC-N2 Sensor. A USB-SPI device was used to overcome these issues.

The HPMA115S0 does not give access to a raw particulate number and was not able to capture variations of particulate matter at levels of concentrations experienced during the deployment. The time resolution of the OPC-N2 cannot be lower than $2 \mathrm{~s}$, sending commands more frequently creates communication issues, which is exacerbated by the length of the communication wires. For the OPC-N2 and the HPMA115S0, a time resolution of $6 \mathrm{~s}$ was used which correspond to the maximum response time of the HPMA115S0. For the Plantower PMS5003 and PMS7003, the resolution alternates between 1 and 3 seconds depending on the particulate count. The Plantower PMS7003 is connected to the FTDI Chip by a PCB connector board supplied with the sensors. This board has caused intermittent connection issues and needs to be replaced by a direct connection to the sensor pins.

The PM sensors are enclosed in an IP65 ABS enclosure 360x200x160mm (HxWxD) mounted in a portrait orientation. In order to take representative samples the sensors need a constant air flow. This was achieved by drilling a series of $8 \mathrm{~mm}$ holes in the base and side of the enclosure. In order to reduce debris and biological material entering the enclosure these holes are protected by a $3 \mathrm{~mm}$ hole diameter mesh, $1.5 \mathrm{~mm}$ thick and $51 \%$ of open area. The sensors are then mounted on a bulkhead inserted across the width of the box as shown in Figure 2. The Raspberry Pi is mounted above the bulkhead in order to provide additional protection from water ingress. Two of the sensors (PMS5003 \& PMS7003) have the intake and exhaust on the same side. The HPMA115S0 and OPC-N2 exhaust from the opposite side to the intake. This means these sensors are exhausting into the main Raspberry Pi compartment. In order to eliminate the affect of any heat produced by the Raspberry $\mathrm{Pi}$, this exhaust is then ducted away from the enclosure, via a $20 \mathrm{~mm}$ pipe to prevent air 
TABLE I

Main Characteristics of the FAn ASSisted Particulate Matter (PM) SENSORS USED IN the DePloyment

\begin{tabular}{|c|c|c|c|c|c|c|c|}
\hline Model & $\begin{array}{c}\text { Size } \\
(\mathrm{mm}) \\
(\mathrm{HxWxD})\end{array}$ & $\begin{array}{l}\text { Price } \\
\text { (USD) }\end{array}$ & Interface & Current requirement & $\begin{array}{l}\text { Detection } \\
\text { range }(\mu \mathrm{m})\end{array}$ & $\begin{array}{l}\text { Concentration range of } \\
\text { measurement }\left(\mu \mathrm{g} / \mathrm{m}^{3}\right)\end{array}$ & $\begin{array}{l}\text { Raw } \\
\text { output }\end{array}$ \\
\hline Alphasense OPC-N2[22] & $60 \times 64 \times 75$ & 443 & SPI & 175mA@5V DC & 0.38 to 17 & 0.01 to 1,500 & Yes \\
\hline Plantower PMS5003[23] & $38 \times 21 \times 50$ & 28 & UART & 100mA@5V DC & 0.3 to 10 & 0 to 500 & Yes \\
\hline Plantower PMS7003[24] & $37 \times 12 \times 48$ & 28 & UART & 100mA@5V DC & 0.3 to 10 & 0 to 500 & Yes \\
\hline Honeywell HPMA115S0[25] & $36 \times 43 \times 24$ & 33 & UART & 80mA@5V DC & Not known & 0 to 1,000 & No \\
\hline
\end{tabular}

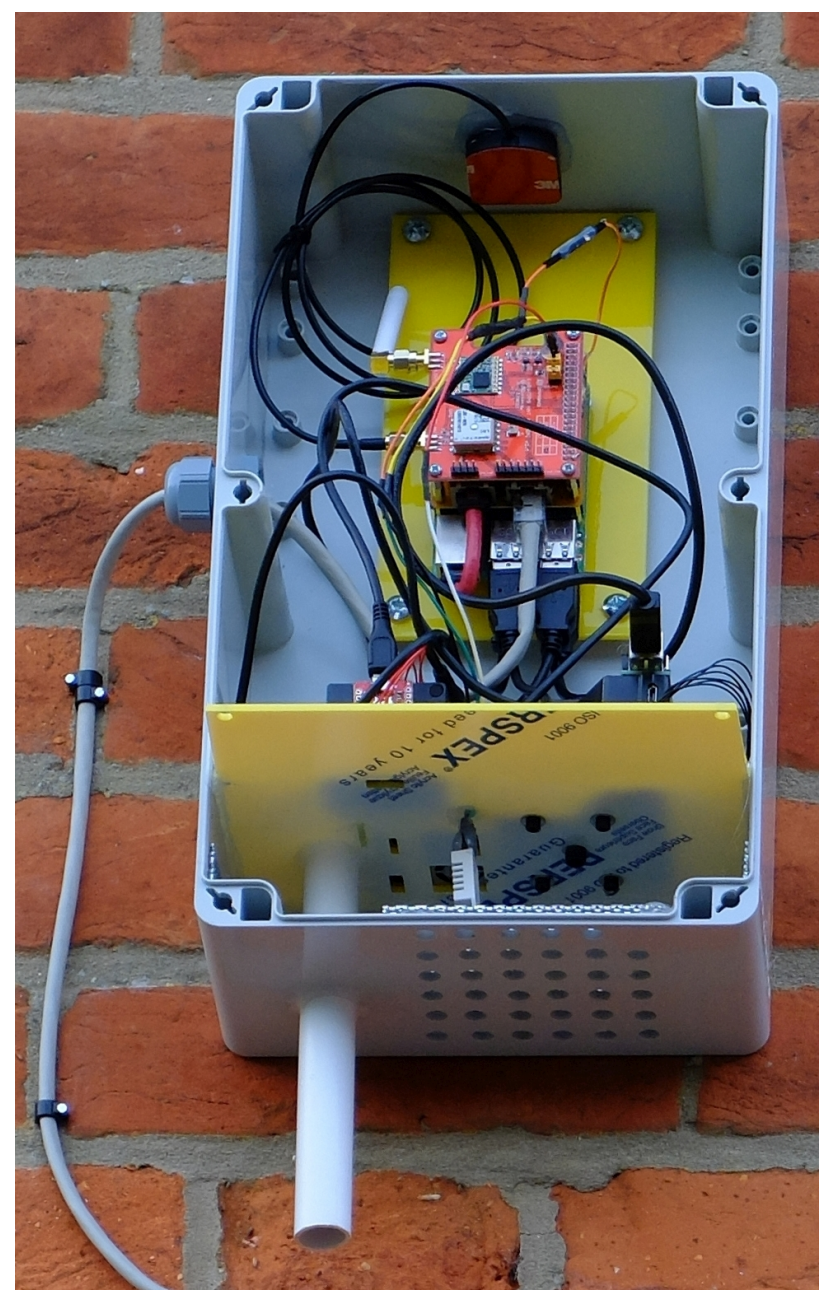

Fig. 2. Air Quality IoT device deployed on an external wall

recirculation. Figure 2 shows the complete Air Quality IoT device deployed on an external wall located at School A, (see Figure 3 for school location).

\section{B. Software}

When using a Raspberry Pi as the processor for a remote deployment careful consideration needs to be given to the Operating System (OS). This software is a single point of failure for the deployment. In this deployment there were conflicting constraints, pragmatism meant that the OS chosen was the latest version of Raspbian Lite. This was chosen because of the ease of development and deployment. This decision was made knowing there are major weaknesses in this choice. When running the $\mathrm{Pi}$ on an unreliable power supply there is a strong possibility of SD corruption unless mitigating steps are taken. The easiest way to protect against this corruption is to run a read-only root file system, this reduces the likelihood of a write being performed when power is lost. It is these failed writes that can cause the corruption.

Another issue with the default Raspbian (Lite) image is the number of pre-installed packages, although making development faster has two main disadvantages: the image is very large, and it increases the number of updates that need deploying. If the device is connected to a high bandwidth network this is not an issue, this is not also the deployment situation faced. Addressing the issues discussed in this section is on-going work and is further discussed in section VII.

\section{Deployment}

Six AQ IoT devices have been deployed across two School sites, shown in Figure 3 within the city of Southampton. At each school the deployment positions have been chosen so that the devices are positioned around the perimeter, with at least one of the devices being located next to a road influenced by school traffic. School A is the closer of the two sites to the AURN monitoring station "Southampton Centre". The AQ IoT devices have been mounted on exterior walls/fences or railings $\approx 2-3 \mathrm{~m}$ high. A single Cat-5 network cable provides power and where possible network from inside the attached building.

\section{Management}

We use Icinga [30] to provide automatic status monitoring of the deployment as it provides failure notification and failure timing, allowing downtime patterns to be identified. A particular consideration when choosing which monitoring software to use is the ability to write custom checks using nagios plugins[31] to enable monitoring of custom aspects of this deployment. We use Microsoft Azure hosted server to host the OpenVPN, Icinga and to store all the data received via rsync from the AQ IoT devices.

\section{DATA CONNECTIVITY}

This deployment aims to provide real-time air quality monitoring data, therefore data connectivity is required in order to enable remote connections to the sensor nodes. Two main technologies have been investigated: OpenVPN[32] and SSH tunnels. The two separate methods of remote access have been chosen to provide resilience and flexibility, to overcome factors outside of the researchers control. Both the OpenVPN and the 


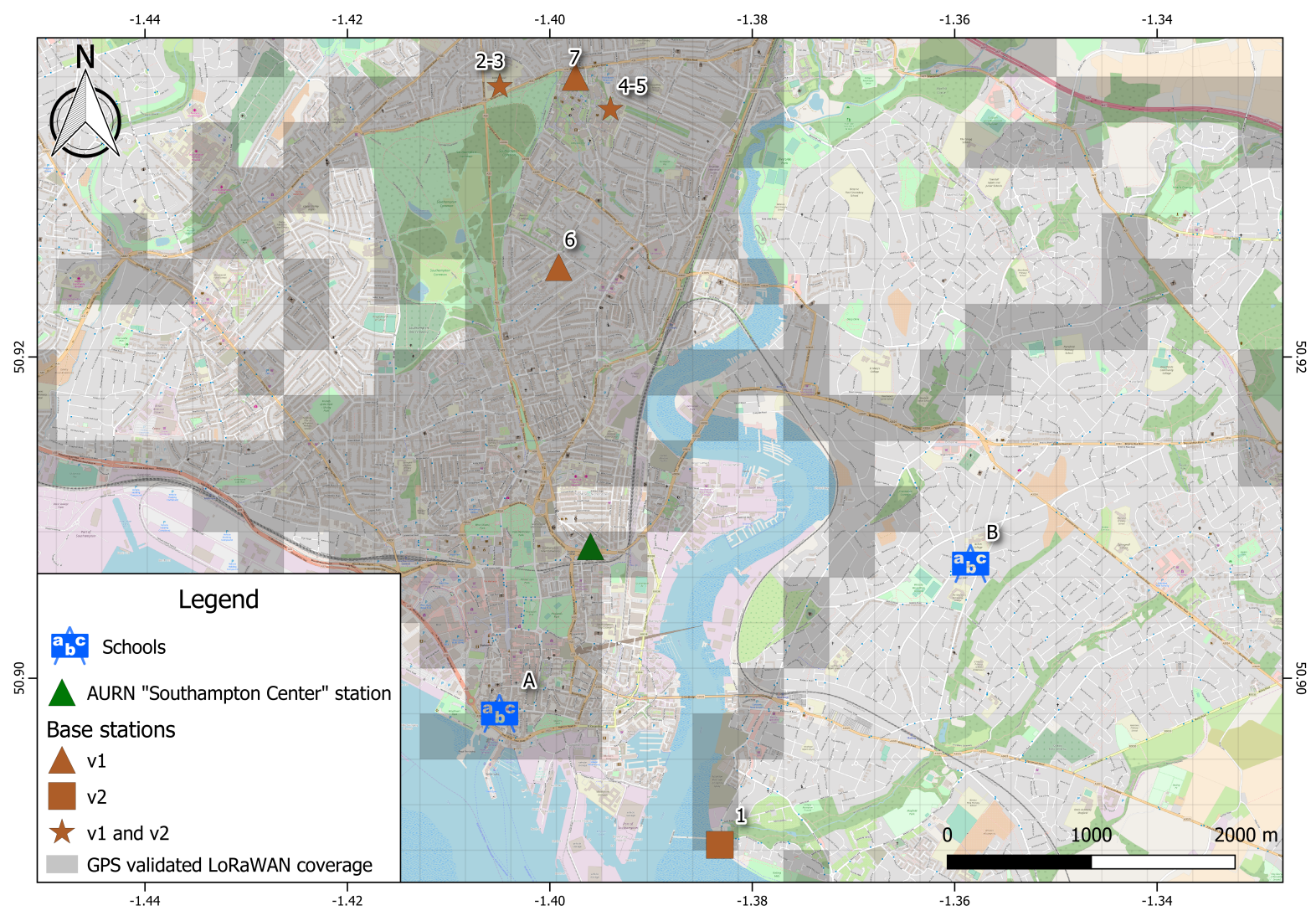

Fig. 3. The 6 deployed Air Quality IoT devices at schools A \& B, the 7 LoRaWAN base stations, and confirmed coverage across Southampton, UK[29].

TABLE II

LORAWAN BANDWIDTH CALCULATED AT $1 \%$ DUTY CYCLE

\begin{tabular}{lrrr} 
Configuration & $\begin{array}{r}\text { Data Rate } \\
\text { (bit/s) }\end{array}$ & $\begin{array}{r}\text { Max Payload } \\
\text { (byte) }\end{array}$ & $\begin{array}{r}\text { Max Data Transfer } \\
\text { (byte/h) }\end{array}$ \\
\hline SF12/125kHz & 250 & 51 & 1,065 \\
SF11/125kHz & 440 & 51 & 1,947 \\
SF10/125kHz & 980 & 51 & 4,359 \\
SF9/125kHz & 1,760 & 115 & 7,877 \\
SF8/125kHz & 3,125 & 222 & 14,032 \\
SF7/125kHz & 5,470 & 222 & 24,582
\end{tabular}

SSH tunnels connect from the IoT device to a Linux VPS running on the Microsoft Azure cloud platform. The OpenVPN tunnel allows the IoT device to be accessed directly from the cloud server. The SSH tunnel that the IoT device establishes to the server initiates a reverse tunnel enabling users on the server to connect into the device.

Not all of our AQ IoT devices will have wired/wireless network connectivity so we have included a LoRaWAN module to allow low bandwidth long range communication [33], see Table II. The aim is to validate using LoRaWAN as the sole communication channel. We have deployed a total of 6 base stations in Southampton and a $7^{\text {th }}$ has been installed by a $3^{\text {rd }}$ party, as shown in Table III and Figure 3.

\section{RESUlts}

A first analysis of the data collected over a period of 12 days between the $8^{t h}-20^{t h}$ of February by one of the IoT devices in School A revealed that while the Alphasense OPC-N2 and the Plantower PMS5003 and PMS7003 were able to capture variations in PM concentrations, the Honeywell HPMA115S0 showed little to no variations in the PM concentration in any of the boxes. We suspect that the Honeywell HPMA115S0 was below its detection limit. The variations of PM concentration measured by the other sensors show similar trends to the variations measured by the respective sensors of the two other AQ IoT devices located at School A.

The closest AURN monitoring station, "Southampton Centre", is located about $1 \mathrm{~km}$ away from School A (see Figure 3). This station produces hourly $\mathrm{PM}_{2.5}$ concentration data that we compared to the $\mathrm{PM}_{2.5}$ concentrations measured by the sensors of the AQ IoT devices and averaged by hour. We also compared it with the $\mathrm{PM}_{2.5}$ concentrations measured captured during the same period of time by one of the IoT device located at School B. The data for $\mathrm{PM}_{2.5}$ concentrations from the AURN station are not yet available for the $13^{t h}-16^{\text {th }}$ February. Table IV gives the Root Mean Square Error (RMSE) and the Pearson coefficient $\left(\mathrm{R}^{2}\right)$ of the sensor data compared when using "Southampton centre" monitoring station as a reference using a sensor evaluation toolbox[34]. The Plantower 
TABLE III

LORAWAN BASE STATIONS FOR SOUTHAMPTON CITY

\begin{tabular}{rlrlllc} 
& Name & Altitude $(\mathrm{m})$ & Brand & Model & Antenna & $3^{\text {rd }}$ Party \\
\hline 1 & 7276FFFFFE10292 & 8 & Kerlink & iBTS & Procom CXL 900-3LW-NB (Dual) & No \\
2 & 7276FFFFFE103EC & 85 & Kerlink & iBTS & Procom CXL 900-3LW/I & No \\
3 & B827EBFFFEE36EF8 & 85 & Raspberry Pi & IMST iC880A & Procom CXL 900-3LW-NB & No \\
4 & 7276FFFFFE103F0 & 50 & Kerlink & iBTS & Procom CXL 900-3LW/I & No \\
5 & B827EBFFFE2D3798 & 45 & Raspberry Pi & IMST iC880A & Taoglas OMB & No \\
6 & B827EBFFFE71AB02 & 65 & Raspberry Pi & IMST iC880A & Taoglas OMB & No \\
7 & B827EBFFFEAC4B12 & 60 & Raspberry Pi & IMST iC880A & RF Solutions FLEXI-SMA90-868 & Yes
\end{tabular}

TABLE IV

Root Mean Square Error and Pearson coefficient of one Iot DEVICE AT EACH SCHOOL

\begin{tabular}{lllll} 
& \multicolumn{2}{c}{ School A } & \multicolumn{2}{c}{ School B } \\
Sensor & RMSE & $\mathrm{R}^{2}$ & RMSE & $\mathrm{R}^{2}$ \\
\hline Alphasense OPC-N2 & 0.052 & 0.276 & 0.045 & 0.259 \\
Plantower PMS5003 & 0.030 & 0.694 & 0.024 & 0.577 \\
Plantower PMS7003 & 0.027 & 0.669 & 0.024 & 0.566 \\
Honeywell HPMA115S0 & 0.044 & 0 & 0.038 & 0
\end{tabular}

PMS5003 and PMS7003 obtain significantly better RMSE and $\mathrm{R}^{2}$ than the OPC-N2 and HPMA115S0.

\section{CONCLUSION AND FUTURE WORK}

We conclude that low-cost PM micro-sensors are viable and intend to perform a larger deployment to investigate further. Our deployed AQ IoT Devices show that not all PM sensors are equal but that it is possible to achieve a good correlation with the AURN stations. Further work is required to determine (i) how sensor operation varies according to changes in environmental characteristics, (ii) how sensor data tracks reference monitoring station data in co-localisation studies, and (iii) whether sensor data from such boxes can be corrected to provide sufficiently reliable data where reference stations are not viable and where improved spatio-temporal resolution of PM concentrations is required.

With careful selection of hardware and data transmission optimisation it is possible to operate a LoRaWAN based sensor network and remain with in the $1 \%$ duty cycle. Using LoRaWAN has proven invaluable as a secondary channel of communication, providing hourly readings even during network outages and at locations without network access. Although all the raw data is stored on the AQ IoT Devices, the LoRaWAN messages confirm device operation, reducing the need for physical site visits. Spreading Factor 10 proved a good trade-off between airtime (and hence duty cycle limits) and transmission range. Table II shows that this will facilitate a total of $4.2 \mathrm{kbit}$ of data payload per hour. By optimising our binary data storage, selecting only the data channels required (e.g. PM2.5) and limiting to two PM sensors we believe that it is possible to use LoRaWAN to transmit all our sensor data. This opens up new opportunities for sensor location as we only require power and LoRaWAN coverage. As part on ongoing future work we intend to experiment with using LoRaWAN only devices and potentially alternative sources of power. The overall aim is to gain a better spread of sensor devices across the city.
Regardless of the network connectivity we are experimenting with using LoRaWAN as a means to control the AQ IoT Devices as the message sizes are suitably small and can be queued for delivery for example, to perform maintenance, e.g. Reboot, request rebuild or update (via wired network) and reconfigure sensor polling rates.

Three of our LoRaWAN base stations are Kerlink iBTS devices which support LoRaWAN v2.0 [35] one of the features that this offers is location of the transmit message, without the need for GPS. One of our goals is to make the AQ IoT devices mobile/portable and a key limitation is power requirements. Eliminating GPS and WiFi drastically reduces power requirements, the Raspberry $\mathrm{Pi}$ has lower power hardware variants and OS optimisations can assist with reducing overall powder consumption. We are currently seeing LoRaWAN receive ranges of over $12 \mathrm{~km}$ using SF7, but the coverage is not uniform. We have over 25, 000 GPS validated LoRaWAN data points across the city. The tested LoRaWAN coverage is shown in Figure 3 and online at ttnmapper.org.

As discussed in Section IV-B the default Raspbian OS has some limitations, mainly its inability to recover from SD card corruptions and a difficulty with full operating system updates (especially fail-over and recovery). We recognised this as a limitation early on and built a custom minimal Linux distribution for use in subsequent deployments.

The custom-built minimal Linux distribution has been built using the Yocto project tools [36]. We mainly decided to follow this route due to the flexibility it offers around choosing only the necessary packages, libraries and binaries. Most of the popular Single Board Computer (SBC) Operating Systems (i.e. Raspbian) are trimmed down versions of PC distributions, which although valuable for beginners and hobbyists, are too large for resources constrained field deployments. Power failure can cause SD card corruptions on read/write partitions, so we used the Yocto tools to make the operating system partitions read-only.

The custom Operating System has four major components: Over the Air (OTA) updates capabilities (OSTree) [37], containerisation (Docker) [38] and the tools required for the management infrastructure. OTA capabilities allow us to add new packages and make changes to the OS for example, security updates. OSTree was chosen because it sends updates in the form of deltas. It only sends OS differences as opposed to other tools of this type that send full OS updates requiring larger bandwidth and could be difficult to deploy in remote locations. OSTree benefits from open source management 
tools, such as the GENIVI [39], built to ease the management of OTA deployments.

Containerisation offers a new perspective over deploying and managing applications. The custom-built OS runs applications in Docker containers, which allows us to remotely add stop, start and delete applications within containers. One of the most important advantage containerisation offers is ease of building applications independent of the host OS, eliminating dependency problems and enabling portability. It offers fault tolerance and security capabilities, and management tooling such as Cockpit [40], which allows us to manage Docker containers and OS updates.

The underlying datasets can be found at: https://doi.org/10.5281/zenodo.1217023

\section{ACKNOWLEDGMENTS}

We acknowledge funding from the following sources: Engineering and Physical Sciences Research Council; Next Generation Unmanned Systems Science Centre for Doctoral Training supported by the Natural Environmental Research Council [NE/L002531/1]; the Leverhulme Trust through the Southampton Marine and Maritime Institute; Engineering and Physical Sciences Research Council [EP/P004024/1]; Matthew Loxham is supported by a BBSRC Future Leader Fellowship [BB/P011365/1]. Southampton City Council and two local schools supported this pilot study. Richard Lansdowne and Semtech for their ongoing LoRaWAN support.

\section{REFERENCES}

[1] W. Jiao, G. Hagler, R. Williams et al., "Community Air Sensor Network (CAIRSENSE) project: Evaluation of low-cost sensor performance in a suburban environment in the southeastern United States," Atmospheric Measurement Techniques, vol. 9, no. 11, pp. 5281-5292, 2016.

[2] D. Hasenfratz, O. Saukh, C. Walser et al., "Deriving high-resolution urban air pollution maps using mobile sensor nodes," Pervasive and Mobile Computing, vol. 16, no. PB, pp. 268-285, 2015.

[3] N. Castell, M. Kobernus, H. Y. Liu et al., "Mobile technologies and services for environmental monitoring: The Citi-Sense-MOB approach," Urban Climate, vol. 14, pp. 370-382, December 2015.

[4] COMEAP, The Mortality Effects of Long-Term Exposure to Particulate Air Pollution in the United Kingdom, 2010.

[5] P. J. Landrigan, R. Fuller, N. J. Acosta et al., "The Lancet Commission on pollution and health," October 2017.

[6] J. S. Apte, K. P. Messier, S. Gani et al., "High-Resolution Air Pollution Mapping with Google Street View Cars: Exploiting Big Data," Environmental Science and Technology, vol. 51, no. 12, pp. 6999-7008, 2017.

[7] D. Fecht, A. L. Hansell, D. Morley et al., "Spatial and temporal associations of road traffic noise and air pollution in London: Implications for epidemiological studies," Environment International, vol. 88, pp. 235242, 2016.

[8] H. Lin, T. Liu, J. Xiao et al., "Hourly peak PM2.5 concentration associated with increased cardiovascular mortality in Guangzhou, China,' Journal of Exposure Science and Environmental Epidemiology, vol. 27, no. 3, pp. 333-338, May 2017

[9] K.-H. Kim, E. Kabir, and S. Kabir, "A review on the human health impact of airborne particulate matter," Environment International, vol. 74, pp. 136-143, January 2015.

[10] A. Y. Watson, R. R. Bates, and D. Kennedy, Air Pollution, the Automobile, and Public Health, 1988, vol. 704, no. 10.

[11] Air Quality Expert Group, "Particulate Matter in the United Kingdom," Tech. Rep., 2005.

[12] P. Schneider, N. Castell, M. Vogt et al., "Mapping urban air quality in near real-time using observations from low-cost sensors and model information," Environment International, vol. 106, pp. 234-247, 2017.
[13] J. J. West, A. Cohen, F. Dentener et al., "What We Breathe Impacts Our Health: Improving Understanding of the Link between Air Pollution and Health," Environmental Science \& Technology, vol. 50, no. 10, pp. 4895-4904, May 2016.

[14] J. Li, B. Faltings, O. Saukh et al., "Sensing the Air We BreatheThe OpenSense Zurich Dataset." in Conference of Association for the Advancement of Artificial Intelligence, 2012, pp. 323-325.

[15] J. Van den Bossche, J. Peters, J. Verwaeren et al., "Mobile monitoring for mapping spatial variation in urban air quality: Development and validation of a methodology based on an extensive dataset," Atmospheric Environment, vol. 105, pp. 148-161, 2015.

[16] J. Van den Bossche, J. Theunis, B. Elen et al., "Opportunistic mobile air pollution monitoring: A case study with city wardens in Antwerp," Atmospheric Environment, pp. 408-421, 2016.

[17] E. G. Snyder, T. H. Watkins, P. A. Solomon et al., "The Changing Paradigm of Air Pollution Monitoring," Environmental Science \& Technology, vol. 47, no. 20, pp. 11369-11377, 2013.

[18] K. A. Koehler and T. M. Peters, "New Methods for Personal Exposure Monitoring for Airborne Particles," pp. 399-411, December 2015.

[19] World Health Organization, "WHO Air quality guidelines for particulate matter, ozone, nitrogen dioxide and sulfur dioxide: global update 2005: summary of risk assessment," Geneva: World Health Organization, pp. 1-22, 2006.

[20] L. Spinelle, M. Gerboles, M. G. Villani et al., "Field calibration of a cluster of low-cost available sensors for air quality monitoring. Part A: Ozone and nitrogen dioxide," in Sensors and Actuators, B: Chemical, vol. 215, 2015, pp. 249-257.

[21] A. C. Rai, P. Kumar, F. Pilla et al., "End-user perspective of low-cost sensors for outdoor air pollution monitoring," pp. 691-705, December 2017.

[22] Alphasense Ltd, "Alphasense user manual opc-n2 optical particle counter," 2015. [Online]. Available: https://goo.gl/D7nKaF

[23] Z. Yong, "Digital universal particle concentration sensor pms5003 series data manual," 2016. [Online]. Available: https://goo.gl/MnH8hi

[24] _ - "Digital universal particle concentration sensor pms7003 series data manual," 2016. [Online]. Available: https://goo.g1/25914C

[25] Honeywell International Inc., "Hpm series particle sensor," 2017. [Online]. Available: https://goo.gl/RcHxxo

[26] D. J. Law, A. Healey, P. Anslow et al., "IEEE Std 802.3-2015," 2016. [Online]. Available: http://ieeexplore.ieee.org/document/7428776/

[27] L. R. Crilley, M. Shaw, R. Pound et al., "Evaluation of a low-cost optical particle counter (Alphasense OPC-N2) for ambient air monitoring," Atmospheric Measurement Techniques Discussions, no. August, pp. 124, 2017.

[28] R. Williams, A. Kaufman, T. Hanley et al., "Evaluation of field-deployed low cost PM sensors," no. December, 2014.

[29] “OpenStreetMap." [Online]. Available: https://www.openstreetmap.org/ copyright

[30] "Icinga." [Online]. Available: https://www.icinga.com/

[31] "Nagios plugins." [Online]. Available: https://www.nagios.org/projects/ nagios-plugins/

[32] J. Yonan, 2002. [Online]. Available: http://openvpn.net

[33] A. J. Wixted, P. Kinnaird, H. Larijani et al., "Evaluation of LoRa and LoRaWAN for wireless sensor networks," in 2016 IEEE SENSORS. IEEE, oct 2016, pp. 1-3.

[34] B. Fishbain, U. Lerner, N. Castell et al., "An evaluation tool kit of air quality micro-sensing units," Science of the Total Environment, vol. 575, no. September 2016, pp. 639-648, jan 2016.

[35] LoRa Alliance, "Lorawan Geolocation Whitepaper," 2018. [Online]. Available: https://docs.wixstatic.com/ugd/ecccla d43b3b29dfff4ec2b00f349ced4225c4.pdf

[36] O. Salvador and D. Angolini, Embedded Linux Development with Yocto Project. Packt Publishing Ltd, 2014.

[37] "Ostree." [Online]. Available: https://ostree.readthedocs.io/

[38] J. Rufino, M. Alam, J. Ferreira et al., "Orchestration of containerized microservices for iiot using docker," in Industrial Technology (ICIT), 2017 IEEE International Conference on. IEEE, 2017, pp. 1532-1536.

[39] Wind River Systems, "Autonomous cars from a unique perspective: Reality," 2016.

[40] “Cockpit project.” [Online]. Available: http://cockpit-project.org/ 